\title{
Apoptogenic activity of a synthetic cantharimide in leukaemia: Implication on its structural activity relationship
}

\author{
STANTON HON LUNG KOK ${ }^{1}$, CHUNG HIN CHUI ${ }^{1,2}$, WING SZE LAM ${ }^{1}$, JIEN CHEN $^{1}$, FUNG YI LAU ${ }^{2}$, \\ RAYMOND SIU MING WONG ${ }^{2}$, GREGORY YIN MING CHENG ${ }^{2}$, WING KA TANG ${ }^{1}$, IVY TUANG NGO TEO ${ }^{1}$, \\ FILLY CHEUNG ${ }^{1}$, CHOR HING CHENG ${ }^{1}$, ALBERT SUN CHI CHAN ${ }^{1}$ and JOHNNY CHEUK ON TANG ${ }^{1}$ \\ ${ }^{1}$ Cancer Drug Research and Development Laboratory, Central Laboratory of the Institute of Molecular Technology for Drug \\ Discovery and Synthesis, State Key Laboratory of Chinese Medicine and Molecular Pharmacology, Department of Applied \\ Biology and Chemical Technology, The Hong Kong Polytechnic University; ${ }^{2}$ Department of Medicine and Therapeutics, \\ Prince of Wales Hospital, The Chinese University of Hong Kong, P.R. China
}

Received June 26, 2006; Accepted September 4, 2006

\begin{abstract}
Cantharidin isolated from Mylabris caraganae and other insects has been used as an anti-cancer drug in China for many years. However, its toxicity on the renal system and suppression effect on bone marrow limits its usage clinically. A synthetic analogue of cantharidin (CAN 037) has been shown to have cytotoxic effect on the SK-Hep 1 hepatoma cell line but its underlying working principle remains undefined. Here we further report the action of CAN 037 on an acute myelogenous leukaemia (AML) cell line, KG1a. [3-(4,5dimethylthiazol-2-yl)-5-(3-carboxymethoxyphenyl)-2-(4sulfophenyl)-2H-tetrazolium] (MTS) assay was used to demonstrate the cytotoxicity of CAN 037 on KG1a cells. Morphological changes of CAN 037-treated leukaemia cells were recorded under an inverted microscope. Possible activation of caspase 3,8 and 9 from KG1a cells was also investigated. KG1a AML cells were sensitive to CAN 037. Morphological changes including cell shrinkage and loss of colony formation ability were observed. Caspase 3, 8 and 9 activity was elevated, whereas pre-incubating the KG1a cells with the generic caspase inhibitor z-VAD-fmk could only partially reverse the CAN 037-induced cell death. In addition to the SK-Hep-1 hepatoma cell line, CAN 037 is also effective in inducing the death of KG1a AML cells in vitro. Apoptosis is involved in the action of CAN 037 including the activation of the caspase family. Caspase-dependent cell death pathway may be necessary but not essential in CAN 037-induced apoptosis of KG1a cells. Further consideration of the structural
\end{abstract}

Correspondence to: Dr Johnny Cheuk On Tang, Department of Applied Biology and Chemical Technology, The Hong Kong Polytechnic University, Hung Hom, Hong Kong, P.R. China

E-mail: bccotang@inet.polyu.edu.hk

Key words: cantharimide, leukaemia, structural activity relationship activity relationship of CAN 037 may provide opportunities to improve its therapeutic value.

\section{Introduction}

Plants are very important and useful sources of molecules of great interest for the development of new pharmaceutical products (1). Many of them are found to be effective in cancer therapy but are considerably expensive. Examples include the isolation of taxol from Taxus brevifolia and both vincristine and vinblastin from Catharenthus roseus. It is estimated that the unit price of taxol is now approximately US\$ 600000 per $\mathrm{kg}$ while the unit price of both vincristine and vinblastin could be up to US\$ 1000 000-2 000000 per kg (2).

Recently, we have demonstrated the possible in vitro underlying working principles of many Traditional Chinese medicines (TCMs) in herbal form extract that have been claimed to yield anti-cancer potential (3), including the fruit of Gleditsia sinensis (4), Scutellaria barbata and Radix Sophorae Tonkinensis (5) as well as Brucea javanica (6). Our results bring TCMs closer to a scientific approach.

Cantharidin has been used for the clinical treatment of tumours including hepatocellular carcinoma and leukaemia. It can be isolated naturally from many types of commonly found insects including the dried body of Mylabris caraganae. However, cantharidin is highly toxic (7). Therefore, many modified cantharidin analogues are synthesized chemically in order to achieve a comparable anti-tumour property to cantharidin but with a less toxic effect on non-tumour cells.

Recently, Lin et al published a family of cantharidin analogues which showed cytotoxic effect on hepatoma cells but no further mechanistic work followed (8). We have studied the underlying working principle of one of them [compound 3e (8), which we name CAN 037: Fig. 1] using the KG1a acute myelogenous leukaemia (AML) model. Our results demonstrated that CAN 037 showed certain selectivity between the KG1a AML cell line and non-malignant haematological disordered bone marrow. Furthermore, its chemical structure provides a skeleton for chemical modification. 


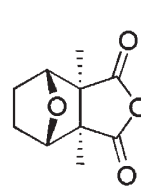

Cantharidin

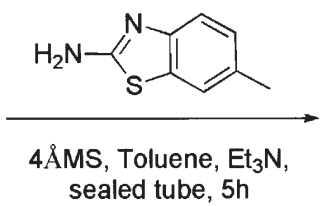

sealed tube, $5 \mathrm{~h}$

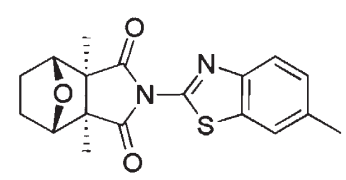

CAN037
Figure 1. Chemical synthesis scheme of CAN 037.

\section{Materials and methods}

Synthesis of CAN 037. The chemical synthesis of CAN 037 was detailed previously (8). Nuclear magnetic resonance (NMR) was further performed to confirm the structure of CAN 037.

Cell lines and cell culture. The human AML cell line KG1a was obtained from American Type of Culture Collection. It was maintained in minimum essential medium (JRH Biosciences) supplemented with $10 \%$ of heat inactivated fetal bovine serum (Hyclone) together with antibiotics involving penicillin and streptomycin. Cells were allowed to grow in a humidified cell culture incubator with $5 \% \mathrm{CO}_{2}$.

Human bone marrow cell collection and isolation. Nonmalignant haematological disorder bone marrow cells were collected from consenting patients by bone marrow aspirate method. A total of three adult subjects were recruited. Immediately after collection, mononuclear cells were enriched by Ficoll Plaque (General Electricity, Amersham) gradient centrifugation. Cells were washed twice with phosphatebuffered saline and resuspended in complete medium. Viable cell percentage was then estimated by trypan blue exclusion assay and counted using a haemacytometer as mentioned.

[3-(4,5-dimethylthiazol-2-yl)-5-(3-carboxymethoxyphenyl)-2(4-sulfophenyl)-2H-tetrazolium] (MTS) assay. Changes in the cellular viability of CAN 037-treated cells were monitored using the MTS activity assay as reported previously. Briefly, KG1a and non-malignant haematological disorder bone marrow cells were seeded at day 0. After 6 h, CAN 037 was added at different concentrations. After $48 \mathrm{~h}$ of incubation, the medium was removed and MTS/PMS solution was added and the cells were incubated for a further $30 \mathrm{~min}$ exactly. Afterwards, optical absorbance was determined at $490 \mathrm{~nm}$ according to the user manual (Promega).

Morphological monitoring of CAN 037-treated KG1a cells. Any morphological changes in CAN 037-treated KG1a leukaemia cells were recorded by investigation under an inverted microscope at the scheduled time point.

Caspase activity assay. To study whether caspase 3, 8 and 9 protease activity was increased after $24 \mathrm{~h}$ of CAN 037 treatment of KG1a AML cells, we used time resolved fluorescence technology based caspase 3 and 8 activity kits purchased from Lance Perkin Elmer (Finland) while caspase 9 activity was assayed by the Promega luminescence kit. Briefly, CAN 037treated cells were washed with PBS and then total cellular protein extracts were prepared and quantitated by the Bradford method using bovine serum albumin as calibration. Afterwards, caspase 3, 8 or 9 specific substrate and detection buffer were added to an equal amount of total cancer cell protein extract and individual caspase activity was analysed according to the instruction manuals provided. Finally, the resulting relative fluorescence units or relative luminscence units were compared with the untreated control.

Caspase dependency cytotoxicity assay. To determine whether CAN 037-mediated induction of apoptosis involved also caspase-independent pathways, KG1a AML cells were pretreated with caspase inhibitor $\mathrm{z}-\mathrm{VAD}$-fmk, at a concentration of $20 \mu \mathrm{M}$. Afterwards, CAN 037 was added and cancer cells were further incubated for $48 \mathrm{~h}$. The resulting cellular viability was then quantified by using the MTS assay as mentioned previously.

\section{Results}

Synthesis of CAN 037. We followed the chemical reaction procedure published by Lin et al and the structure of CAN 037 was confirmed by NMR analysis (Figs. 1 and 2 respectively). The compound was dissolved in DMSO in a concentration of $25 \mathrm{mg} / \mathrm{ml}$ and stored at $-20^{\circ} \mathrm{C}$ before use. Since the maximum concentration of CAN 037 tested was $\leq 25 \mu \mathrm{g} / \mathrm{ml}$, the contribution of solvent was always $<0.1 \%$.

Cytotoxicity of CAN 037 on the KGla AML cell line and nonmalignant haematological disorder bone marrow. To see whether CAN 037 could be also cytotoxic to KG1a AML cells and primary culture of human bone marrow cells, we tested it by using MTS/PMS assay. After $48 \mathrm{~h}$ of incubation, a dosedependent cytotoxic relationship was observed. The MTS50 (the concentration of CAN 037 required to reduce $50 \%$ of the MTS/PMS reduction activity of incubated cells when compared with the untreated control) was found to be between 12.5 and $25 \mu \mathrm{g} / \mathrm{ml}$ (Fig. 3A). Morphologically, there was also a dosedependent pattern where extensive cell shrinkage was clearly observed when CAN 037 was at $25 \mu \mathrm{g} / \mathrm{ml}$ after $48 \mathrm{~h}$. Interestingly, even the mean MTS activity of KG1a cells at a concentration of $12.5 \mu \mathrm{g} / \mathrm{ml}$ of CAN 037 was as high as $80 \%$ when compared with the untreated control, no colony formation was observed. This suggested that the clonogenicity potential of KG1a was being readily affected, even cell survival was preserved at $12.5 \mu \mathrm{g} / \mathrm{ml}$ of CAN 037 (Fig. 3B-D). All of the three non-malignant haematological disorder bone marrow samples showed $70-80 \%$ of MTS activity between 12.5 and $25 \mu \mathrm{g} / \mathrm{ml}$ (data not shown). Our results suggested that there were certain degrees of growth inhibitory selectivity for CAN 037 on KG1a leukaemia cells and primary culture of bone marrow samples respectively.

Mechanistic action of CAN 037 on KGla AML cells. To demonstrate whether caspase-dependent or -independent pathway is involved in CAN 037-induced KG1a apoptosis, we examined the caspase 3,8 and 9 activity of KG1a protein extract after CAN 037 incubation. Fig. 4 demonstrates that the activation of all of these caspases was recruited but to different extents. Therefore, we conclude that intrinsic programmed cell 


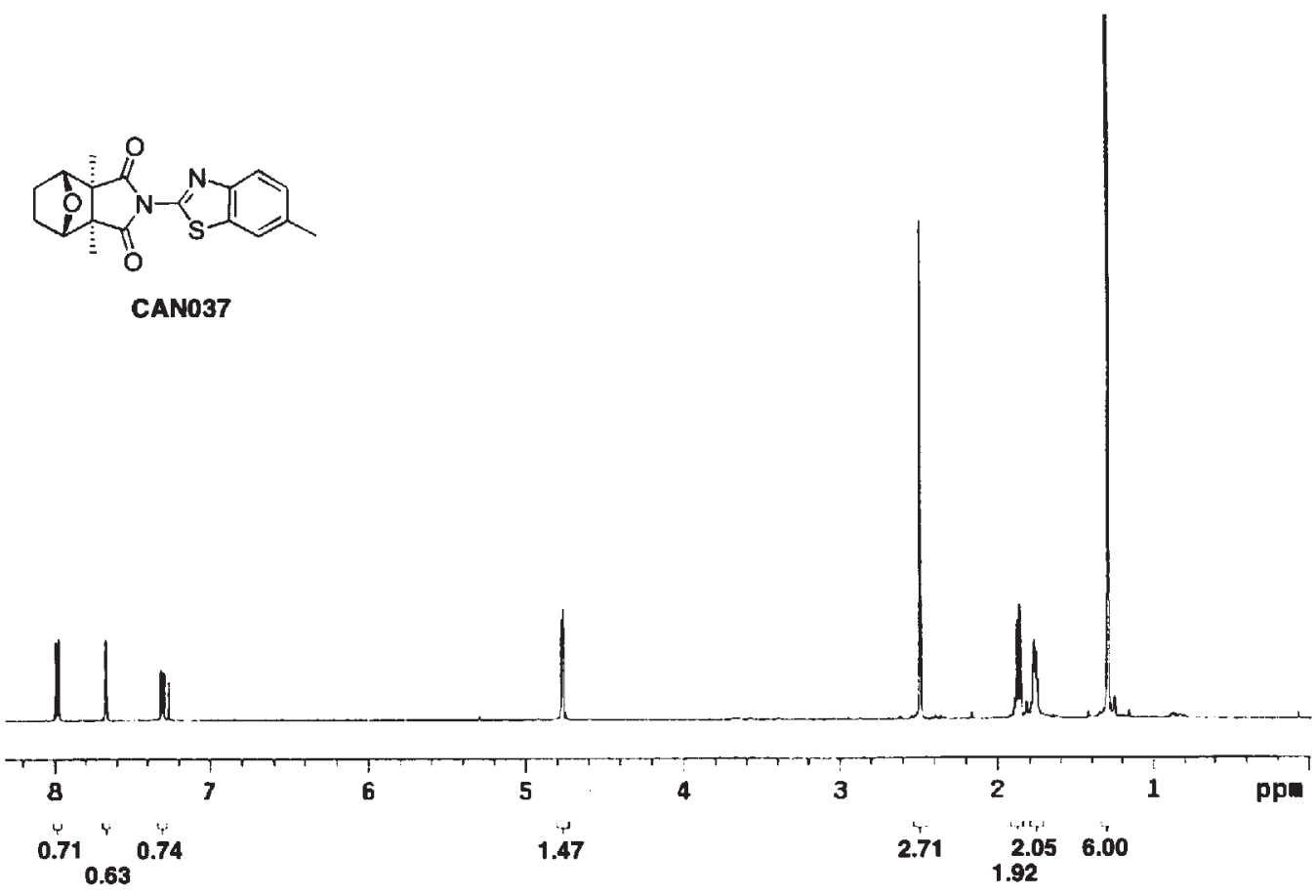

Figure 2. ${ }^{1} \mathrm{H}$ spectrum of CAN 037.
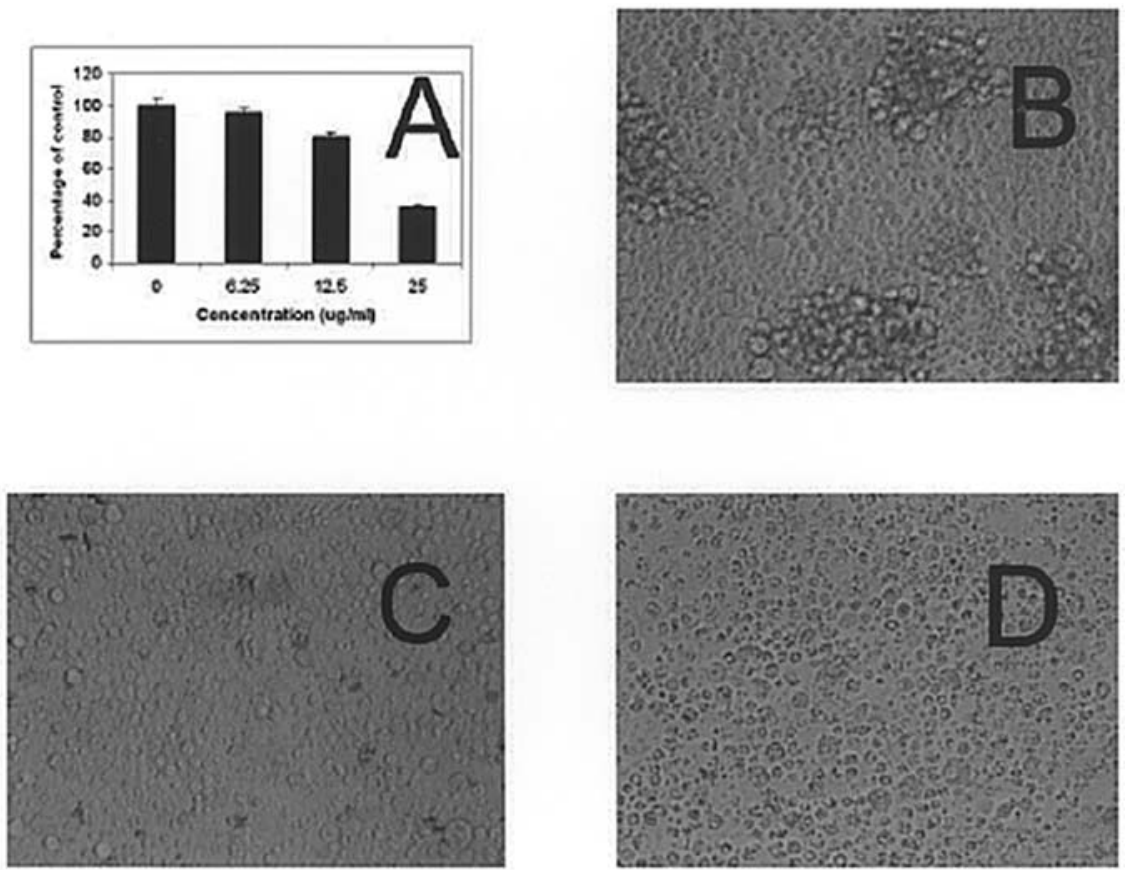

Figure 3. (A) Dose response relationship for the cytotoxic activity of CAN 037 on KG1a AML cells after 48 h of incubation. Morphological demonstration of KG1a cells under the influence of CAN 037. (B) KG1a cells incubated with $0.1 \%$ DMSO as the vehicle control. (C) KG1a cells incubated with $12.5 \mu \mathrm{g} / \mathrm{ml}$ of CAN 037. (D) KG1a cells incubated with $25 \mu \mathrm{g} / \mathrm{ml}$ of CAN 037.

death pathway is highly likely to be involved in CAN 037induced apoptosis of KG1a cells.

Is caspase dependent pathway exclusively required in CAN 037-dependent cell death? If caspase 3,8 and 9 are activated during CAN 037-induced apoptosis, we questioned whether inhibition of all of these caspases could completely inhibit the cytotoxic action of CAN 037. To approach this hypothesis, the KG1a cells were pre-incubated with the generic caspase inhibitor z-VAD-fmk so that caspase activity could be significantly inhibited in the presence of CAN 037 (Fig. 4). As shown in Fig. 5, the MTS activity of KG1a cells increased only slightly in the presence of CAN 037 when they were pre-incubated with $\mathrm{z}-\mathrm{VAD}$-fmk. This phenomenon showed 


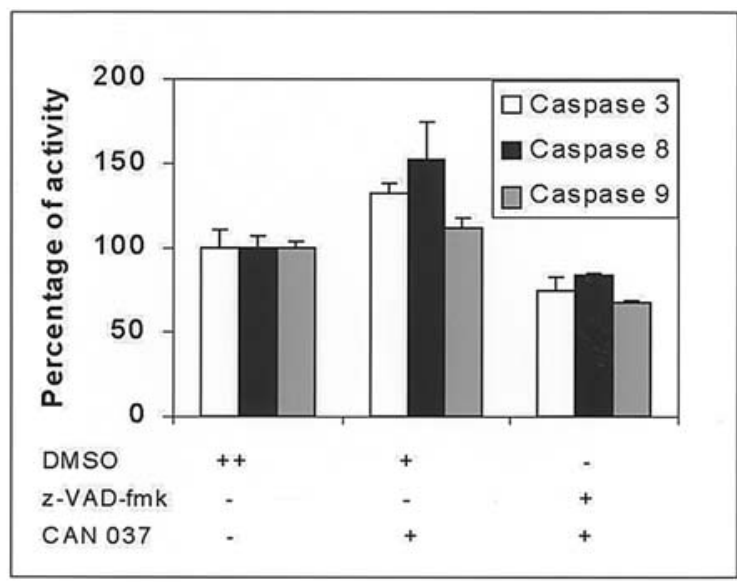

Figure 4. Caspase activity analysis from KG1a AML cells after $48 \mathrm{~h}$ of incubation with CAN 037 at $25 \mu \mathrm{g} / \mathrm{ml}$. Details of the experiment are presented in Materials and methods. Each plus sign indicates a contribution of $1 \mu 1$ per $1000 \mu 1$ of the total volume. Results represent the average \pm SD of three independent experiments.

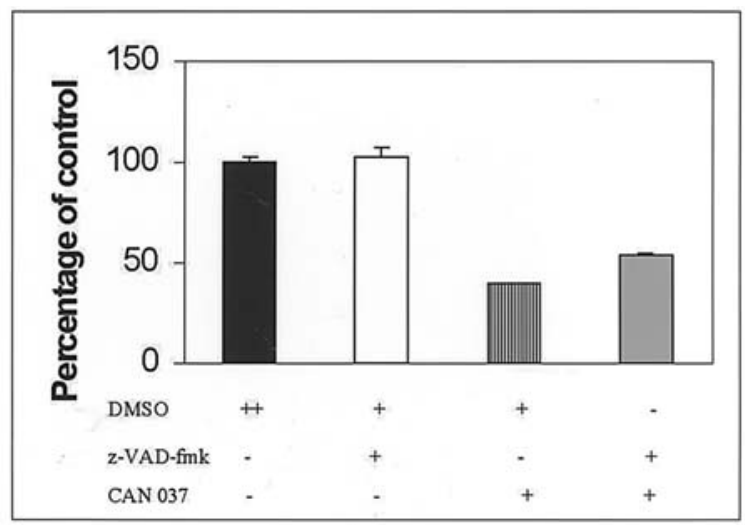

Figure 5. Cytotoxicity assay on CAN 037 -treated KG1a cells after $48 \mathrm{~h}$ of incubation in the absence or presence of the caspase inhibitor z-VAD-fmk. Each experiment was performed in triplicate. Each plus sign indicates a contribution of $1 \mu 1$ per $1000 \mu 1$ of the total volume. Results represent the average \pm SD of three independent experiments.

that caspase activity seemed to be necessary but might not be essential for CAN 037-induced apoptosis of KG1a AML cells and it is speculated that both caspase-dependent and -independent pathways are active in vitro.

\section{Discussion}

Based on the principle chemical structure of cantharidin, we have synthesized a number of its family analogues and named them the CAN series. After obtaining a family of cantharidin analogues, we further screened its possible cytotoxic activity using a panel of carcinoma cell lines. Two of these synthetic samples (CAN 029 and CAN 030) showed a significant cytotoxic response on cancer cell lines. However, they also exhibited similar cytotoxicity on non-malignant haematological disorder bone marrow (9) in vitro.

We then further moved to synthesize another cantharidin analogue named CAN 032 (10). CAN 032 showed a certain level of selectivity for the primary culture of non-malignant

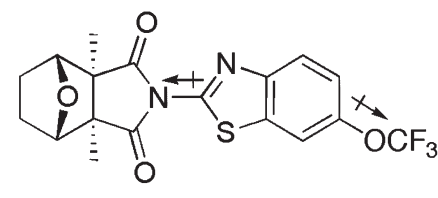

CAN032

Figure 6. Graphic representation for the proposed electron distribution condition on the compound CAN 032

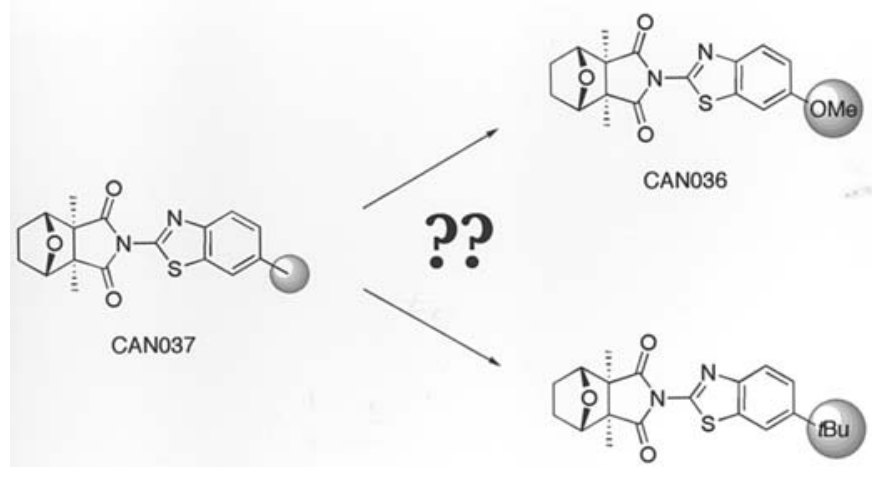

Figure 7. Proposed chemical modification scheme on the compound CAN 037. Two derivatives or analogues will be synthesized for cytotoxicity screening on both carcinoma cells and non-malignant haematological disorder bone marrow primary culture.

haematological disorder bone marrow and hepatoma cells. We also demonstrated the underlying working principle of CAN 032 by using KG1a AML cells as the demonstration model (11). CAN 032 thus provided a milestone for further chemical synthesis of cantharidin analogues.

When referring back to the chemical structure of CAN 032, we noticed that there is an interesting electron distribution of the molecule (Fig. 6). As the core structure of cantharidimide with diimide and the 6-position trifluoromethoxy group are both electron withdrawing groups, this would make the benzothiazole moiety of CAN 032 under a high electron deficiency state. We speculate that this special electron distribution status may exert a crucial effect on its cytotoxicity to carcinoma cells.

Similarly, Lin et al also stated that the electronegativity of the substituent group plays an important role in the cytotoxicity (8). However, they assumed that the increase of electronegativity of the substituent group would decrease the cytotoxic nature for the benzothiazole derivatives. The chemical structure of CAN 037 opens a new modification opportunity by modifying the methyl group at the 6-position. We hypothesize that, based on the results of CAN 032 and CAN 037, one important characteristic of cantharidin is that its relative reactivity toward anti-cancer activity may be controlled by the introduction of an electronic withdrawing or donating group at the 6-position. Currently, we are studying both hypotheses by either changing the methyl group at the 6-position of CAN 037 into a more electron donating group or a more electron withdrawing group (Fig. 7). The resulting cytotoxicity on cancer cells will clarify which assumption is applicable. 
Recently, there have been many reports on the novel synthesis of cantharidin-related compounds (12) as well as their possible molecular action in cancer cells (13). Here we demonstrated that the activated biological pathways of CAN 037-stimulated cancer cells in vitro are similar to those of CAN 029, CAN 030 and CAN 032. The cross pollination of biological and chemical concept allows us to have a better understanding of the structural activity relationship of cantharidin analogues during anti-cancer drug synthesis. This could be implicated in synthesizing a novel anti-tumour drug under a cost-effective pathway in the future.

\section{Acknowledgement}

We acknowledge the support by the Area of Strategic Development (ASD) programme of the Hong Kong Polytechnic University (H.K.P.U.) (work programme A012). This study was supported by the Areas of Excellence Scheme established under the University Grants Committee of the Hong Kong Special Administrative Region, P.R. China (project no. AoE/P-10/01) and a Niche Area grant offered by the H.K.P.U. Drs F.Y. Lau and C.H. Chui are the honourary tutors kindly offered by Professors J.J.Y. Sung and G.Y.M. Cheng from the Department of Medicine and Therapeutics, Prince of Wales Hospital, The Chinese University of Hong Kong. Drs S.H.L. Kok and C.H. Chui are supported by the post of 'Research Fellow' kindly offered by Professor A.S.C. Chan. Dr S.H.L. Kok would like to thank Dr C.H. Chui who gave valuable advice to his research team on the anti-cancer drug screening experiments.

\section{References}

1. Khan MTH, Ather A and Gambari R: The role of metabolic engineering for the production of secondary metabolites of plants. Minerva Biotec 17: 127-131, 2005.

2. Rao SR and Ravishankar GA: Plant cell cultures: chemical factories of secondary metabolites. Biotech Adv 20: 101-153, 2002.
3. Chui CH, Gambari R, Lau FY, Teo ITN, Ho KP, Cheng GYM, Ke B, Higa T, Kok SHL, Chan ASC and Tang JCO: Anti-cancer potential of traditional Chinese herbal medicines and microbial fermentation products. Minerva Biotec 17: 183-191, 2005.

4. Chui CH, Lau FY, Chan ASC, Cheng GYM, Wong RSM, Lai KB, Kok SHL, Au Yeung TTL, Teo ITN, Yau MYC, Cheung F, Cheng $\mathrm{CH}$ and Tang JCO: Gleditsia sinensis fruit extractinduced apoptosis involves changes of reactive oxygen species level, mitochondrial membrane depolarization and caspase 3 activation. Int J Mol Med 15: 539-543, 2005.

5. Chui CH, Lau FY, Tang JCO, Kam KL, Cheng GYM, Wong RSM, Kok SHL, Lai PBS, Ho R, Gambari R and Chan ASC: Activities of fresh juice of Scutellaria barbata and warmed water extract of Radix Sophorae Tonkinensis on antiproliferation and apoptosis of human cancer cell lines. Int J Mol Med 16: 337-341, 2005

6. Lau FY, Chui CH, Gambari R, Kok SHL, Kan KL, Cheng GYM, Wong RSM, Teo ITN, Cheng CH, Wan TSK, Chan ASC and Tang JCO: Antiproliferative and apoptosis-inducing activity of Brucea javanica extract on human carcinoma cells. Int J Mol Med 16: 1157-1162, 2005.

7. Tsauer W, Lin JG, Lin PY, Hsu FL and Chiang HC: The effects of cantharidin analogues on xanthine oxidase. Anticancer Res 17: 2095-2098, 1997.

8. Lin PY, Shi SJ, Shu HL, Chen HF, Lin CC, Liu PC and Wang LF: A simple procedure for preparation of $N$-Thiazoyl and $N$ Thiadiazolycantharidinimides and evaluation of their cytotoxicities against human hepatocellular carcinoma cells. Bioorganic Chem 28: 266-272, 2000.

9. Kok SHL, Chui CH, Lam WS, Chen J, Tang JCO, Lau FY, Cheng GYM, Wong RSM and Chan ASC: Induction of apoptosis on carcinoma cells by two synthetic cantharidin analogues. Int $\mathrm{J}$ Mol Med 17: 151-157, 2006.

10. Kok SHL, Chui CH, Lam WS, Chen J, Lau FY, Cheng GYM, Wong RSM, Lai PBS, Leung TWT, Tang JCO and Chan ASC: Apoptotic activity of a novel synthetic cantharidin analogue on hepatoma cell lines. Int J Mol Med 17: 945-949, 2006.

11. Kok SHL, Chui CH, Lam WS, Chen J, Lau FY, Wong RSM, Cheng GYM, Tang WK, Cheng CH, Tang JCO and Chan ASC: Mechanistic insight into a novel synthetic cantharidin analogue in a leukaemia model. Int J Mol Med 18: 375-379, 2006.

12. Yu CW, Li KK, Pang SK, Au Yeung SC and Ho YP: Anticancer activity of a series of platinum complexes integrating demethylcantharidin with isomers of 1,2-diaminocyclohexane. Bioorg Med Chem Lett 16: 1686-1691, 2006.

13. Li JL, Cai YC, Liu XH and Xian LJ: Norcantharidin inhibits DNA replication and induces apoptosis with the cleavage of initiation protein Cdc6 in HL-60 cells. Anticancer Drugs 17: 307-314, 2006. 